Swarthmore College

Works

Physics \& Astronomy Faculty Works

Physics \& Astronomy

7-1-2007

\title{
Periodic Accretion From A Circumbinary Disk In The Young Binary UZ Tau E
}

Eric L.N. Jensen

Swarthmore College, ejensen1@swarthmore.edu

Saurav Dhital , '06

K. G. Stassun

J. Patience

W. Herbst

See next page for additional authors

Follow this and additional works at: https://works.swarthmore.edu/fac-physics

Part of the Astrophysics and Astronomy Commons

Let us know how access to these works benefits you

\section{Recommended Citation}

Eric L.N. Jensen; Saurav Dhital , '06; K. G. Stassun; J. Patience; W. Herbst; F. M. Walter; M. Simon; and G. Basri. (2007). "Periodic Accretion From A Circumbinary Disk In The Young Binary UZ Tau E". Astronomical Journal. Volume 134, Issue 1. 241-251. DOI: 10.1086/518408

https://works.swarthmore.edu/fac-physics/9

This work is brought to you for free by Swarthmore College Libraries' Works. It has been accepted for inclusion in Physics \& Astronomy Faculty Works by an authorized administrator of Works. For more information, please contact myworks@swarthmore.edu. 
Authors

Eric L.N. Jensen; Saurav Dhital , '06; K. G. Stassun; J. Patience; W. Herbst; F. M. Walter; M. Simon; and G. Basri 


\author{
PERIODIC ACCRETION FROM A CIRCUMBINARY DISK IN THE YOUNG BINARY UZ TAU E \\ Eric L. N. Jensen, ${ }^{1}$ Saurav Dhital, ${ }^{1,2}$ Keivan G. Stassun, ${ }^{2}$ Jenny Patience, ${ }^{3}$ William Herbst, ${ }^{4}$ \\ Frederick M. Walter, ${ }^{5}$ Michal Simon, ${ }^{5}$ and Gibor Basri ${ }^{6}$ \\ Received 2007 January 29; accepted 2007 March 22
}

\begin{abstract}
Close pre-main-sequence binary stars are expected to clear central holes in their protoplanetary disks, but the extent to which material can flow from the circumbinary disk across the gap onto the individual circumstellar disks has been unclear. In binaries with eccentric orbits, periodic perturbation of the outer disk is predicted to induce mass flow across the gap, resulting in accretion that varies with the binary period. This accretion may manifest itself observationally as periodic changes in luminosity. Here we present a search for such periodic accretion in the premain-sequence spectroscopic binary UZ Tau E. We present BVRI photometry spanning 3 years; we find that the brightness of UZ Tau E is clearly periodic, with a best-fit period of $19.16 \pm 0.04$ days. This is consistent with the spectroscopic binary period of 19.13 days, refined here from analysis of new and existing radial velocity data. The brightness of UZ Tau E shows significant random variability, but the overall periodic pattern is a broad peak in enhanced brightness, spanning more than half the binary orbital period. The variability of the $\mathrm{H} \alpha$ line is not as clearly periodic, but given the sparseness of the data, some periodic component is not ruled out. The photometric variations are in good agreement with predictions from simulations of binaries with orbital parameters similar to those of UZ Tau E, suggesting that periodic accretion does occur from circumbinary disks, replenishing the inner circumstellar disks and possibly extending the timescale over which they might form planets.
\end{abstract}

Key words: accretion, accretion disks - binaries: spectroscopic — circumstellar matter planetary systems: protoplanetary disks — stars: individual (UZ Tauri E) stars: pre-main-sequence

Online material: color figure

\section{INTRODUCTION}

It is now well established that most stars are members of binary systems at birth and that many of these stars are surrounded by disks similar to those found around young single stars (see, e.g., the recent review by Monin et al. 2007). Thus, understanding the origin of binaries is vital to understanding the star formation process. The predominance of binaries also means that, based on the number of systems alone, most potential sites of planet formation lie in multiple systems. However, interactions between stars and disks in binary systems can alter disk structure (Beckwith et al. 1990; Jensen et al. 1994, 1996a, 1996b; Osterloh \& Beckwith 1995; Jensen \& Mathieu 1997), resulting in a more complicated environment for planet formation. Nonetheless, the discovery of planets in relatively close binary systems (Eggenberger et al. 2004) shows that binary systems are viable sites of planet formation. Understanding the extent to which binaries modify the structure of their surrounding disks is important for understanding the possible diversity of planetary system environments. In addition, although the mass ratios are different, the interactions between stellar binary companions and disks involve the same physics as those between planetary companions and disks (e.g., D'Angelo et al. 2006) but are more easily observable. Thus, an

\footnotetext{
${ }^{1}$ Department of Physics and Astronomy, Swarthmore College, Swarthmore, PA 19081, USA; ejensen1@swarthmore.edu.

${ }^{2}$ Department of Physics and Astronomy, Vanderbilt University, Nashville, TN 37235, USA.

${ }^{3}$ Division of Physics, Mathematics, and Astronomy, California Institute of Technology, Pasadena, CA 91125, USA.

${ }^{4}$ Astronomy Department, Wesleyan University, Middletown, CT 06459, USA.

${ }^{5}$ Department of Physics and Astronomy, State University of New York, Stony Brook, NY 11794-3800, USA.

${ }^{6}$ Astronomy Department, University of California, Berkeley, CA 94720, USA.
}

understanding of binary-disk interactions may help us understand planet formation around single stars as well.

A binary star system may have up to three disks: two circumstellar disks, one each around the primary and secondary, and a circumbinary disk outside the binary orbit. Both analytic calculations and numerical simulations show that the region between these disks is not stable for orbiting disk material. However, the question of how easily material can flow from the outer, circumbinary disk across the gap to the circumstellar disks has not been clear from either an observational or a theoretical standpoint. The spectral energy distributions of some young binaries show the clear signature of a cleared central region, while other, apparently similar systems do not (Jensen \& Mathieu 1997). Theoretical analyses by Lin \& Papaloizou (1993) and Artymowicz $\&$ Lubow (1994) suggested that material in the region around the binary orbit is cleared, creating a quasi-equilibrium structure with three distinct disks and a cleared region between them. If the gap between disks is impermeable, the disks evolve independently of each other. Since the presence of a binary companion may increase the rate of accretion (Clarke 1992; Ostriker et al. 1992) and the binary orbit presents a constraint on the size of each circumstellar disk, the circumstellar disks would be exhausted much more quickly than disks around single stars. However, smoothed particle hydrodynamic simulations by Artymowicz \& Lubow (1996, hereafter AL96; see also Günther \& Kley 2002) predicted that material may indeed flow from the circumbinary disk to the circumstellar environment, with the accretion rate varying with the phase of the binary orbit.

If such periodic accretion occurs in young binaries, it may be detectable observationally by a periodic brightening of the system as the material flowing from the circumbinary disk shocks when it collides with the circumstellar disk(s) or accretes onto 
the stellar surface(s). Observations of the T Tauri spectroscopic binary DQ Tau by Mathieu et al. (1997) showed such brightening, occurring at the binary orbital period. In addition, DQ Tau shows periodic variations in spectral veiling and emission-line intensities with orbital phase (Basri et al. 1997), providing strong support for the broad picture of mass flow across gaps suggested by AL96. However, subsequent searches in other young, short-period binary systems surrounded by disks have yielded mixed results. Alencar et al. (2003) did not find periodic photometric variations in AK Sco, but they did find that the blue wing of the $\mathrm{H} \alpha$ line, and both the blue and red wings of the $\mathrm{H} \beta$ line, vary with the binary orbital period, as does the total $\mathrm{H} \alpha$ equivalent width. V4046 Sgr has shown periodic photometric variations at the binary orbital period (Quast et al. 2000; Mekkaden 2000), although an earlier study did not find such variations (Byrne 1986). Like AK Sco, however, V4046 Sgr does show variations in the equivalent width and shape of Balmer lines as a function of orbital phase (Stempels \& Gahm 2004). No dedicated photometric monitoring of UZ Tau E has been reported in the literature to date, but in previous spectroscopic observations, neither $\mathrm{H} \alpha$ equivalent width nor spectral veiling has shown any obvious dependence on binary orbital phase (Martín et al. 2005).

Motivated by previous observational work and a desire to understand accretion in binary systems, we have undertaken a photometric monitoring campaign for the pre-main-sequence (PMS) spectroscopic binary UZ Tau E. UZ Tau, in the Taurus-Auriga starforming region, was first discovered to be variable by K. Bohlin during a bright outburst in 1921 (Bailey 1921; Bohlin 1923) and identified as a $\sim 3.7^{\prime \prime}$ binary by Joy \& van Biesbroeck (1944), one of the first pre-main-sequence binaries to be identified. Subsequently, both components of the binary were found to be binaries themselves, making this a quadruple system. Simon et al. (1992) and Ghez et al. (1993) identified UZ Tau W as a binary system, and Mathieu et al. (1996) identified UZ Tau E to be a single-lined spectroscopic binary with a 19.1 day period. UZ Tau W, a $0.34^{\prime \prime}$ binary (47.6 AU, assuming a distance of 140 pc to Taurus-Auriga; Kenyon et al. 1994), is separated from UZ Tau E by $3.78^{\prime \prime}$ (530 AU; Simon et al. 1995a). Prato et al. (2002) detected absorption lines of the secondary star in the near-infrared spectrum of UZ Tau E, measuring the mass ratio $M_{2} / M_{1}=0.28 \pm 0.01$. Martín et al. (2005) presented additional radial velocity data for UZ Tau E; they found a binary orbital period of 18.979 days and an eccentricity of 0.14 . UZ Tau E shows strong $\mathrm{H} \alpha$ emission, indicative of ongoing accretion, and strong infrared and millimeter excess emission from circumstellar and circumbinary disks; the circumbinary disk has been resolved at $\lambda=1.3 \mathrm{~mm}$ (Jensen et al. 1996a) and $2.6 \mathrm{~mm}$ (Dutrey et al. 1996) with a size and mass comparable to disks around single stars, showing that the close spectroscopic companion has not significantly decreased the disk mass, in contrast to the 50 AU pair in UZ Tau W, where the presence of a companion at a separation comparable to typical disk sizes has greatly reduced the presence of circumstellar material.

In this paper, we present our new photometric observations, as well as a redetermination of the binary orbital parameters from new and existing radial velocity observations. We then examine periodicities in the data, showing that the photometric data vary at the binary orbital period. Finally, we interpret the results in the context of the model of pulsed accretion in binary systems.

\section{OBSERVATIONS}

\subsection{Photometry}

In order to search for periodic photometric variations, we have obtained new photometry of UZ Tau E. Our photometric obser-
TABLE 1

Observations of UZ Tau E

\begin{tabular}{crcccc}
\hline \hline \multirow{2}{*}{ Telescope } & $\begin{array}{c}\text { Exp. Time } \\
(\mathrm{s})\end{array}$ & Filter(s) & Season & $\begin{array}{c}\text { No. of } \\
\text { Nights }\end{array}$ & $\begin{array}{c}\text { Time Span } \\
\text { (days) }\end{array}$ \\
\hline SMARTS $(1.3 \mathrm{~m}) \ldots . .$. & 5 & BVRI & $2003-2004$ & 63 & 170 \\
& 5 & $B V R I$ & $2005-2006$ & 6 & 42 \\
& 30 & $V R I$ & $2005-2006$ & 9 & 33 \\
VVO (0.6 m)............. & 60 & $I$ & $2004-2005$ & 16 & 126 \\
& 60 & $I$ & $2005-2006$ & 20 & 128 \\
\hline
\end{tabular}

vations were made with the $0.6 \mathrm{~m}$ Perkin Telescope at the Van Vleck Observatory (VVO) at Wesleyan University, and with ANDICAM on the $1.3 \mathrm{~m}$ SMARTS Telescope at CTIO. See Table 1 for details of the observations. The data were reduced using standard techniques.

Since UZ Tau E and UZ Tau W are separated by $3.78^{\prime \prime}$, we sought to minimize contamination of the UZ Tau E photometry by light from UZ Tau W by rejecting images with FWHM greater than 7 pixels, and by using a relatively small (3 pixel radius) photometric aperture. Light curves of UZ Tau E and W show no correlation, indicating that the UZ Tau E photometry is uncontaminated.

We performed differential photometry on UZ Tau E using USNO-B1.0 1158-0057597 as a comparison star. The UZ Tau field is relatively sparse, and in some of the SMARTS images this was the only star that was sufficiently bright to serve as a comparison star, so we used it as the sole comparison in all of our photometry. The star was verified to be nonvariable at the few percent level, showing a standard deviation of 0.02 mag by comparison with several other stars of similar brightness using the wider-field VVO images. The USNO-B1.0 catalog (Monet et al. 2003) gives magnitudes for this star from the Second Palomar Sky Survey of $B=16.39, R=13.75$, and $I=12.8$. Although these filters are not identical to those used in our CCD observations, we adopted these values for the magnitude of the reference star to set the zero point of our light curves. In addition, we adopted $V=14.99$ by noting that the $R-I$ color for the comparison star suggests a spectral type of $\sim \mathrm{M} 0$, and adopting a corresponding $B-V$ color. Adopting these values allows us to determine approximate colors for UZ Tau E, although we caution that the absolute scaling of both the individual magnitudes and the colors is uncertain. The differential photometry and the color changes, which form the basis of our analysis, are both unaffected by this systematic uncertainty in the zero point.

\subsection{Spectroscopy}

We did not acquire new spectra of UZ Tau E solely for this program, but we did make new measurements of a number of spectra taken during the course of other programs. Some of these spectra were kindly supplied by Marcos Huerta. These are echelle spectra from the McDonald observatory spanning 14 nights in 2002 January, with $R=46,000$ and wavelength coverage of 5460-6760 $\AA$. The observations and data reduction are described in detail in Huerta et al. (2005). Additional echelle spectra of UZ Tau E were taken at Keck $(R=31,000)$ with the setup described in Basri \& Reiners (2006), and at Lick $(R=48,000)$ with the setup described in Alencar \& Basri (2000).

We used the spectra from Huerta et al. to measure radial velocities of UZ Tau E. Spectra of the weak-lined T Tauri star V819 Tau were used as a radial velocity standard. By cross-correlating the UZ Tau E spectra against the V819 Tau spectra, we measured heliocentric radial velocities of UZ Tau E, assuming $v_{\text {helio }}=$ $14.4 \pm 1.5 \mathrm{~km} \mathrm{~s}^{-1}$ for V819 Tau (Walter et al. 1988). The resulting 
TABLE 2

Radial Velocities and $\mathrm{H} \alpha$ EW

\begin{tabular}{|c|c|c|}
\hline Julian Date & $\begin{array}{c}v_{\text {helio }} \\
\left(\mathrm{km} \mathrm{s}^{-1}\right)\end{array}$ & $\begin{array}{c}\mathrm{H} \alpha \mathrm{EW}^{\mathrm{a}} \\
(\AA)\end{array}$ \\
\hline $2,450,416.82 .$. & $\ldots$ & 88.4 \\
\hline $2,450,783.93 \ldots \ldots \ldots \ldots \ldots \ldots \ldots$ & $\ldots$ & 42 \\
\hline $2,450,783.94 \ldots \ldots \ldots \ldots \ldots \ldots \ldots \ldots \ldots$ & $\ldots$ & 45.1 \\
\hline 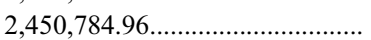 & $\ldots$ & 38 \\
\hline $2,450,785.11 \ldots \ldots \ldots \ldots$ & $\ldots$ & 57 \\
\hline 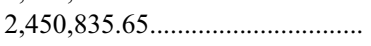 & $\ldots$ & 54: \\
\hline 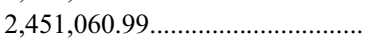 & $\ldots$ & 45.6 \\
\hline 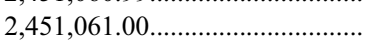 & $\ldots$ & 39.8 \\
\hline 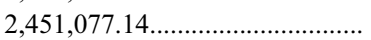 & $\ldots$ & 63.8 \\
\hline $2,451,120.92 \ldots \ldots \ldots \ldots \ldots \ldots \ldots \ldots \ldots \ldots \ldots$ & $\ldots$ & 101 \\
\hline $2,451,137.94 \ldots \ldots \ldots \ldots \ldots \ldots \ldots \ldots \ldots$ & $\ldots$ & 51.3 \\
\hline $2,451,138.88 \ldots \ldots \ldots \ldots \ldots \ldots \ldots \ldots \ldots$ & $\ldots$ & 62.6 \\
\hline $2,451,162.86 \ldots \ldots \ldots \ldots \ldots \ldots \ldots \ldots \ldots \ldots$ & $\ldots$ & 69.3 \\
\hline $2,451,163.82 \ldots \ldots \ldots \ldots \ldots \ldots \ldots \ldots$ & $\ldots$ & 57.8 \\
\hline $2,451,164.79 \ldots \ldots \ldots \ldots \ldots \ldots \ldots \ldots \ldots$ & $\ldots$ & 57.5 \\
\hline 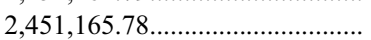 & $\ldots$ & 58.3 \\
\hline 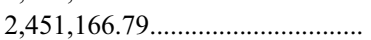 & $\ldots$ & 61.7 \\
\hline 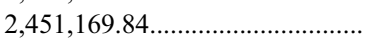 & $\ldots$ & 48.9 \\
\hline 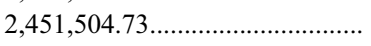 & $\ldots$ & 35 \\
\hline 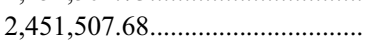 & $\ldots$ & 25: \\
\hline $2,451,508.67 \ldots \ldots \ldots \ldots \ldots \ldots \ldots \ldots \ldots$ & $\ldots$ & 49.1 \\
\hline $2,451,509.71 \ldots \ldots \ldots \ldots \ldots \ldots \ldots \ldots \ldots$ & $\ldots$ & 57 \\
\hline $2,451,510.66 \ldots \ldots \ldots \ldots \ldots \ldots \ldots \ldots \ldots$ & $\ldots$ & 71 \\
\hline 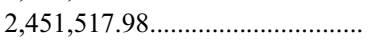 & $\ldots$ & 75 \\
\hline $2,451,523.68 \ldots \ldots \ldots \ldots \ldots \ldots \ldots \ldots$ & $\ldots$ & 42.7 \\
\hline $2,451,524.74 \ldots \ldots \ldots \ldots \ldots \ldots \ldots \ldots \ldots$ & $\ldots$ & 49.3 \\
\hline 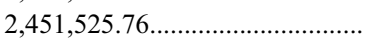 & $\ldots$ & 40.8 \\
\hline 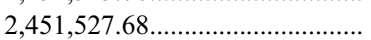 & $\ldots$ & 46.6 \\
\hline 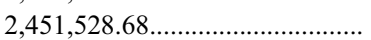 & $\ldots$ & 58.9 \\
\hline 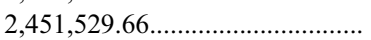 & $\ldots$ & 61.8 \\
\hline $2,451,530.60 \ldots \ldots \ldots \ldots \ldots \ldots \ldots \ldots \ldots$ & $\ldots$ & 69.1 \\
\hline $2,452,280.60 \ldots \ldots \ldots \ldots \ldots \ldots \ldots \ldots \ldots$ & $-4.3 \pm 2.1$ & 44 \\
\hline $2,452,281.74 \ldots \ldots \ldots \ldots \ldots \ldots \ldots \ldots \ldots \ldots \ldots \ldots \ldots \ldots \ldots \ldots$ & $-3.1 \pm 1.5$ & 51 \\
\hline $2,452,282.76 \ldots \ldots \ldots \ldots \ldots \ldots \ldots \ldots$ & $-5.8 \pm 1.8$ & 81 \\
\hline $2,452,283.66 \ldots \ldots \ldots \ldots \ldots \ldots \ldots \ldots \ldots$ & $-4.9 \pm 4.6$ & 78 \\
\hline $2,452,284.71^{\mathrm{b}} \ldots \ldots \ldots \ldots \ldots \ldots \ldots \ldots$ & $\ldots$ & 77 \\
\hline $2,452,286.66 \ldots \ldots \ldots \ldots \ldots \ldots \ldots \ldots \ldots$ & $6.2 \pm 2.6$ & 90 \\
\hline $2,452,287.65 \ldots \ldots \ldots \ldots \ldots \ldots \ldots \ldots \ldots$ & $17.1 \pm 1.7$ & 87 \\
\hline $2,452,288.68 \ldots \ldots \ldots \ldots \ldots \ldots \ldots \ldots \ldots \ldots$ & $27.2 \pm 2.1$ & 59 \\
\hline 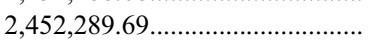 & $29.6 \pm 3.0$ & 65 \\
\hline 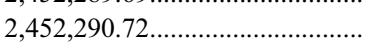 & $37.8 \pm 7.0$ & 67 \\
\hline 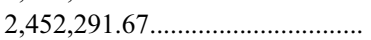 & $28.5 \pm 2.1$ & 58 \\
\hline 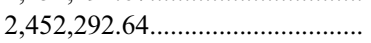 & $29.1 \pm 3.2$ & 50 \\
\hline $2,452,293.67 \ldots \ldots \ldots \ldots \ldots \ldots \ldots \ldots \ldots$ & $22.0 \pm 3.6$ & 45 \\
\hline 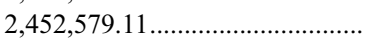 & $\ldots$ & 50 \\
\hline
\end{tabular}

${ }^{a}$ Positive values denote emission.

b The spectrum on this date was too noisy to allow measurement of an accurate radial velocity.

velocities are given in Table 2. Radial velocities were measured using several different echelle orders with strong absorption lines; the quoted uncertainties reflect the dispersion in these different measurements, as well as the uncertainty of V819 Tau's radial velocity.

In addition, we also measured the equivalent width of the $\mathrm{H} \alpha$ line in both sets of spectra in order to track changes in accretion rate over time. Equivalent widths are given in Table 2, with an estimated uncertainty of $10 \%$.

\section{BINARY ORBITAL PARAMETERS}

In order to assess whether or not any periodic photometric variations detected in UZ Tau E are synchronized with the bi-

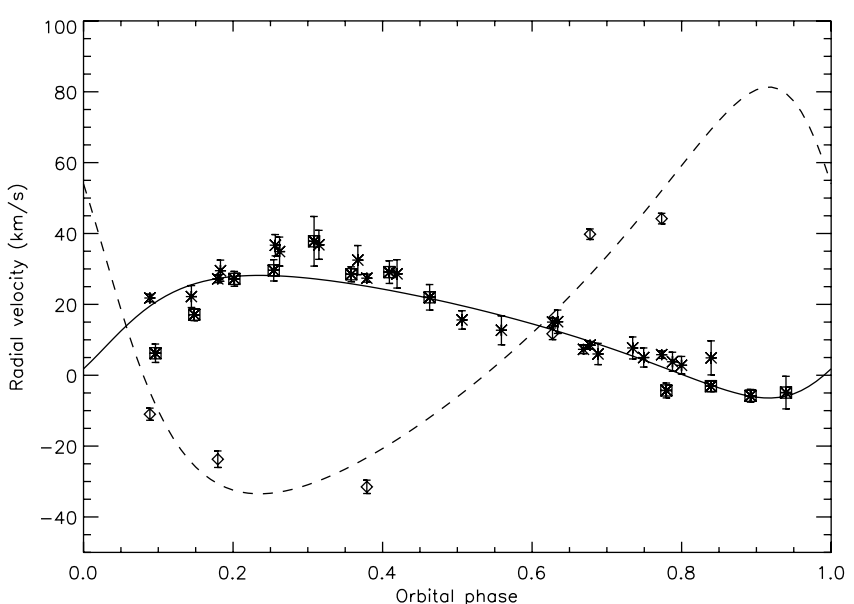

FIG. 1.-Best-fit spectroscopic orbit for UZ Tau E. Asterisks show velocities of the primary; those enclosed in boxes show new radial velocity measurements presented here. Diamonds show velocities of the secondary from Prato et al. (2002). [See the electronic edition of the Journal for a color version of this figure.]

nary orbit, we need to have an accurate knowledge of the orbital parameters. These have been determined previously by Mathieu et al. (1996), Prato et al. (2002), and Martín et al. (2005), but the number of radial velocity points available is still relatively small, especially for the secondary, leaving open the prospect of further improvements to the orbital parameters. To that end, we have reanalyzed the spectroscopic orbit using data published in Prato et al. (2002) and Martín et al. (2005), as well as our new radial velocity measurements $(\S 2.2)$.

We fit the radial velocity data using the Binary Star Combined Solution software (Gudehus 2001), the ORBIT code (Forveille et al. 1999), and our own custom-written IDL code; all gave the same solution. The best-fit phased radial velocity curve is shown in Figure 1, and the orbital parameters are given in Table 3.

The best-fit period of $19.131 \pm 0.003$ days is inconsistent with the value of $18.979 \pm 0.007$ days found by Martín et al. (2005). Examination of the power spectrum of the velocity data used by Martín et al. shows that the 18.979 day period appears to be an alias of the true period, caused by beating of the 19.131 day period with two 6 yr gaps in the radial velocity data; there is a corresponding alias at 19.3 days. Refitting only the data used by Martín et al., we find that the two periods both correspond to local minima in $\chi^{2}$ space, with reduced $\chi^{2}=8.8$ for $P=19.131$ days and reduced $\chi^{2}=10.7$ for $P=18.979$ days. When the new radial velocity data are added, the fit for $P=19.131$ days improves to reduced $\chi^{2}=8.1$ while that for $P=18.979$ days worsens to $\chi^{2}=11.7$, as expected if 19.131 days is the correct period.

TABLE 3

Binary Orbital Parameters for UZ Tau E

\begin{tabular}{|c|c|}
\hline Parameter & Value \\
\hline Period (days) ... & $19.131 \pm 0.003$ \\
\hline & $0.33 \pm 0.04$ \\
\hline 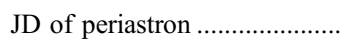 & $2,451,328.3 \pm 0.5$ \\
\hline$\omega(\operatorname{deg})$ & $239 \pm 9$ \\
\hline$a \sin i(\mathrm{AU})$ & $0.124 \pm 0.003$ \\
\hline$\gamma\left(\mathrm{km} \mathrm{s}^{-1}\right)$ & $13.9 \pm 0.7$ \\
\hline$K_{1}\left(\mathrm{~km} \mathrm{~s}^{-1}\right) \ldots \ldots \ldots \ldots \ldots \ldots \ldots$ & $17.3 \pm 1.4$ \\
\hline$K_{2}\left(\mathrm{~km} \mathrm{~s}^{-1}\right) \ldots \ldots \ldots \ldots \ldots \ldots$ & $57.4 \pm 4.7$ \\
\hline$M \sin ^{3} i\left(M_{\odot}\right) \ldots \ldots \ldots \ldots \ldots \ldots \ldots$ & $0.69 \pm 0.13$ \\
\hline$M_{2} / M_{1}$ & $0.30 \pm 0.03$ \\
\hline
\end{tabular}


We note that we have not added any additional radial velocity measurements of the secondary, and thus the mass ratio remains more uncertain than the other orbital elements, resting on the six secondary radial velocities presented by Prato et al. (2002).

$\mathrm{UZ}$ Tau $\mathrm{E}$ is one of only a handful of pre-main-sequence systems with measured stellar masses (see Mathieu et al. [2007] for a recent review). Because the total system mass has been measured (Simon et al. 2000), the spectroscopic orbital parameter $M \sin ^{3} i$ can be used to determine the orbital inclination. This can then be compared with the observed inclination of the circumbinary disk. While this was done by Simon et al. (2000) and Prato et al. (2002), we revisit this issue here using our newly determined orbital parameters for UZ Tau E. Combined with $M=1.31 \pm 0.08 M_{\odot}$ (Simon et al. 2000), our orbital parameters give $\sin i_{\text {orbit }}=0.81 \pm 0.05$, or $i_{\text {orbit }}=54^{\circ} \pm 5^{\circ}$. This is in excellent agreement with the disk inclinations of $54^{\circ} \pm 3^{\circ}$ and $56^{\circ} \pm 2^{\circ}$ measured from interferometric images of the $\lambda=1.3 \mathrm{~mm}$ continuum emission and the $\mathrm{CO}$ line emission, respectively (Simon et al. 2000). Thus, the binary orbit and the circumbinary disk are coplanar. Since the disk inclination is measured at scales of $\sim 100$ AU and the binary orbit is only a few tenths of an astronomical unit, this coplanarity apparently extends over the entire disk. Although there are several theoretical studies of how tilted circumstellar disks interact with a binary system (e.g., Papaloizou \& Terquem 1995; Larwood et al. 1996; Bate et al. 2000; Lubow \& Ogilvie 2000), we know of no studies of the timescale for alignment of a circumbinary disk if it is initially tilted relative to the binary orbit. Studies of the effects of a planetary-mass companion on a tilted external disk (Lubow \& Ogilvie 2001) suggest that such disk tilts do decay over time, however. The example of UZ Tau E shows that coplanarity over the entire disk exists already by an age of a few megayears, suggesting that circumbinary disks either form already aligned with the orbit or come into alignment very quickly. This is similar to the result of Jensen et al. (2004), who found that circumstellar disks in young binaries tend to be aligned with each other, and thus presumably with the binary orbit.

\section{PERIODIC VARIATIONS}

\subsection{Photometry}

In order to determine whether the system is varying in phase with the binary orbit, or in any other systematic way, we have searched the photometric data for periodic signals. We begin by searching for evidence of periodicity, without presupposing a particular period. A Lomb-Scargle periodogram (Scargle 1982) of the I-band data (the band with the largest number of points and best time coverage; Table 1) is shown in Figure 2. There is a strong peak at a period of $19.20 \pm 0.03$ days. The false-alarm probability (FAP) of this peak is less than 0.001 according to the formulation of Horne \& Baliunas (1986). While this FAP calculation is strictly applicable only to evenly spaced data, a Monte Carlo bootstrapping method (e.g., Stassun et al. 1999) confirms that this period is statistically significant at better than $99.9 \%$ confidence. The period uncertainty reported above, which follows from the formulation of Kovacs (1981), is probably underestimated, as it assumes that the underlying signal is well described by a single sinusoid.

Although it is slightly off the main peak of the power spectrum, there is significant power at the binary period of 19.131 days. Periodograms of the $B, V$, and $R$ data are similar (Fig. 3), showing peaks near the binary period, but with broader peaks and higher FAPs, perhaps due to the more limited time coverage of the data in those bands.
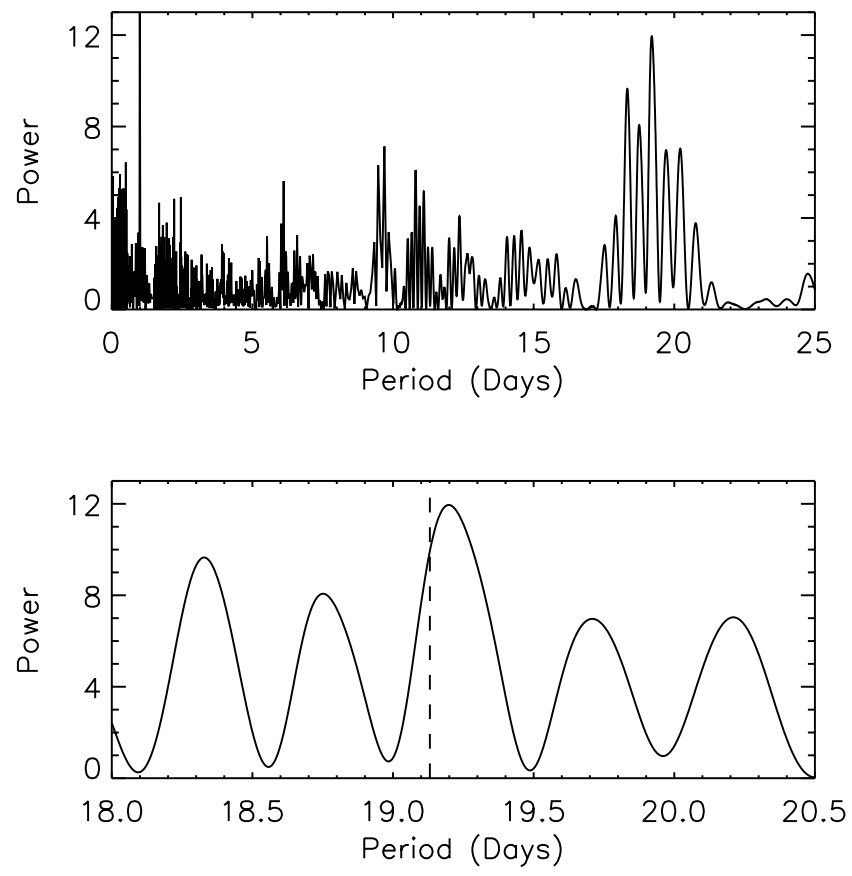

FIG. 2.-Lomb-Scargle periodogram for the $I$-band data. The periodogram peaks at a period of 19.20 days, with a FAP of 0.001 . The dashed line shows the binary orbital period of 19.131 days. The smaller peaks visible flanking the main peak (bottom panel) are near the alias periods expected for beat periods between 1 and 2 yr (caused by the seasonal gaps in the data) and the binary period.

To refine the period and to better estimate its uncertainty, we next performed a phase dispersion minimization (PDM) analysis (Stellingwerf 1978), which is particularly well suited to periodic variability that is highly nonsinusoidal and/or to data with large intrinsic scatter, both of which apply to the photometry of UZ Tau E. The PDM search of the $I$-band data yields a best period of $P=19.17 \pm 0.05$ days, where the uncertainty was determined empirically from the $1 / e$ folding scale of the PDM merit function. The same analysis on the $V$-band data gives $P=19.15 \pm 0.04$ days.

Schwarzenberg-Czerny (1989) argued that a related test, the one-way analysis of variance, is the most powerful statistic of this kind for detection of periodic signals. Applying that test to our data yields $P=19.16 \pm 0.03$ days for the $I$-band data and $P=19.15 \pm 0.05$ days for the $V$-band data. Following Schwarzenberg-Czerny (1989) the period uncertainty was determined using a "postmortem" analysis that measures the $1 \sigma$ confidence interval of the primary periodogram peak, defined by its width at the mean noise power level of the periodogram in the vicinity of this peak. As above, a Monte Carlo permutation analysis of the light curves confirms that this period is statistically significant at better than $99.9 \%$ confidence. Combining these estimates, our best-fit photometric period is $P=$ $19.16 \pm 0.04$ days, consistent with the binary orbital period.

Figure 4 shows $I$-band light curves for all three observing seasons, folded at the binary orbital period of 19.131 days. As suggested by the periodogram analysis, all show indications of periodic behavior, with a broad minimum near orbital phase 0.5 . The data from the 2004-2005 season show the smoothest variability, but this is also the season with the smallest number of data points. Clearly there is significant random variability as well, with scatter of roughly $0.6 \mathrm{mag}$ at all orbital phases.

Figure 5 shows folded $B, V, R$, and $I$ light curves from the 2003-2004 and 2005-2006 seasons. Broadly speaking, the $B V R$ data show the same behavior as the $I$-band light curves, with 

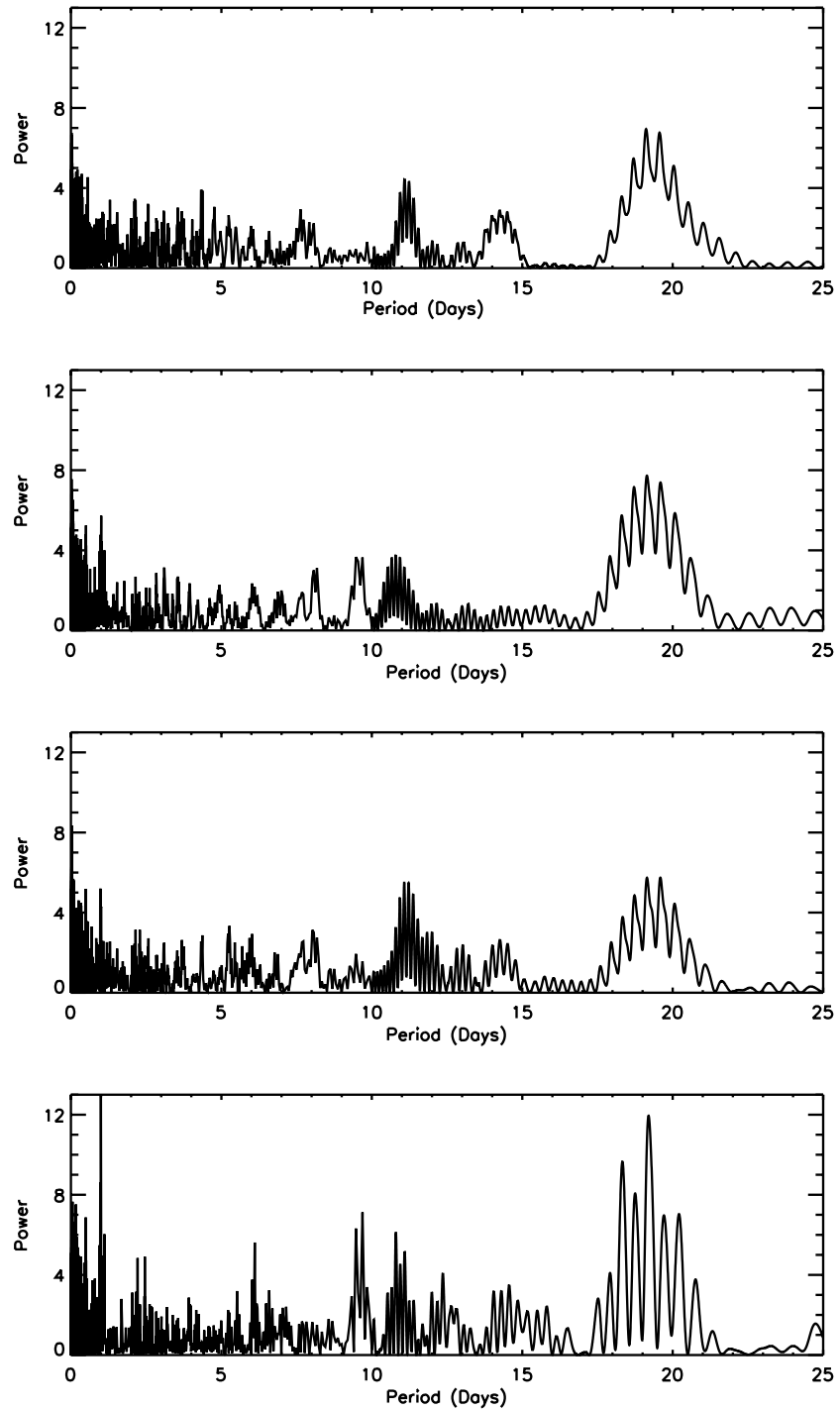

Fig. 3.-Lomb-Scargle periodograms for the $B-, V$-, $R$-, and $I$-band data. All show significant power near the binary orbital period.

large-amplitude variability that appears to have both periodic and random components. We note that the $R$ band includes the $\mathrm{H} \alpha$ line, which may complicate the interpretation of the light curve.

All four bands show a gradual increase in brightness over the 3 years of our observations, with the mean magnitude changing by $0.3 \mathrm{mag}$ at $I$ band from 2003-2004 to 2005-2006. To separate long-term variations from the shorter-term variations of interest here, a linear trend (with a slope of roughly $0.15 \mathrm{mag} \mathrm{yr}^{-1}$ at $I$ band) has been fit to each band. The folded light curves using these detrended data, and combining all three observing seasons, are shown in Figure 6.

In addition to our photometric data, previous data on UZ Tau have shown some evidence of both long-term trends and periodic variations. Bohlin (1923), in one of the first papers to mention UZ Tau, reported on a major flare and then a 4 mag overall decline in brightness from 1921 to 1923 . He also noted that there was a short-period variation with a period of 10-20 days, which encompasses the period of the variations reported here. Bohlin's measurements are for the entire UZ Tau system, but later examination of Bohlin's plates by Herbig (1977) showed that it was UZ Tau E that brightened dramatically in 1921.
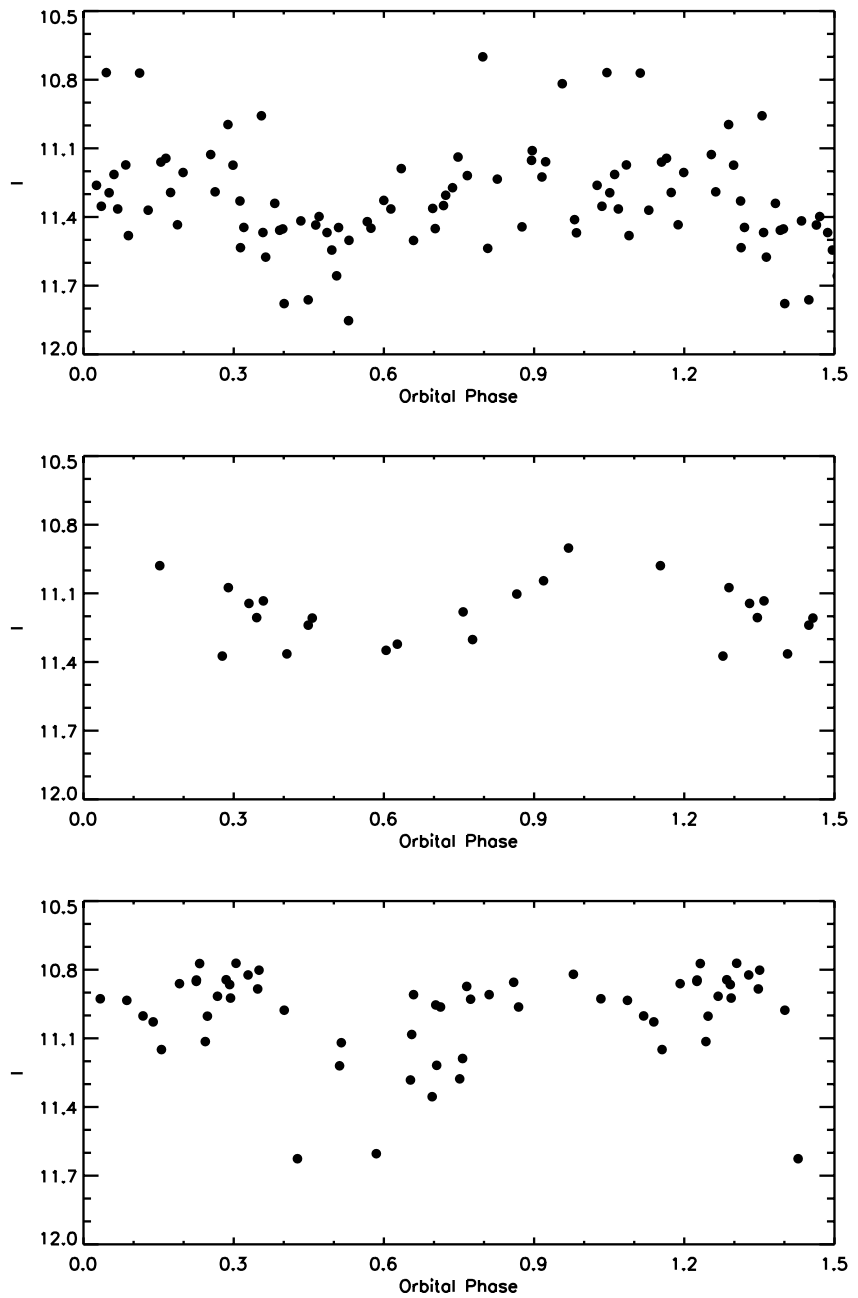

FIG. 4. - I-band magnitude for UZ Tau E folded at the binary orbital period of 19.131 days and plotted against the binary orbital phase. Top to bottom: Data from 2003-2004, 2004-2005, and 2005-2006.

Variations in color of the system can also give clues about the cause of the variability. Figure 7 shows the $V-I$ color as a function of $I$ magnitude and orbital phase. The system shows a behavior commonly seen in T Tauri stars, appearing redder when fainter, and bluer when brighter (Herbst et al. 1994). This behavior is consistent either with periodic changes in extinction (causing both dimming and reddening when the extinction is higher) or in accretion (adding additional blue light when the accretion rate is higher).

\subsection{Spectroscopy}

If the periodic photometric variations are due to changes in accretion rate, one might expect accompanying variations in $\mathrm{H} \alpha$ emission or spectral veiling, common tracers of accretion. Martín et al. (2005) searched for both of these in UZ Tau E and did not find evidence of either. With our new spectra, we can revisit the question of $\mathrm{H} \alpha$ variability.

Figure 8 shows the equivalent width of the $\mathrm{H} \alpha$ emission line as a function of binary orbital phase. The variations do not appear to be strongly correlated with orbital phase. There is some evidence for lower $\mathrm{H} \alpha$ equivalent widths around phase $0.4-0.8$, as seen in the photometric data, but the data are relatively sparse in that phase range as well.

While a lack of periodic variability would be at odds with the photometric data, we note that periodicity may not be as obvious 

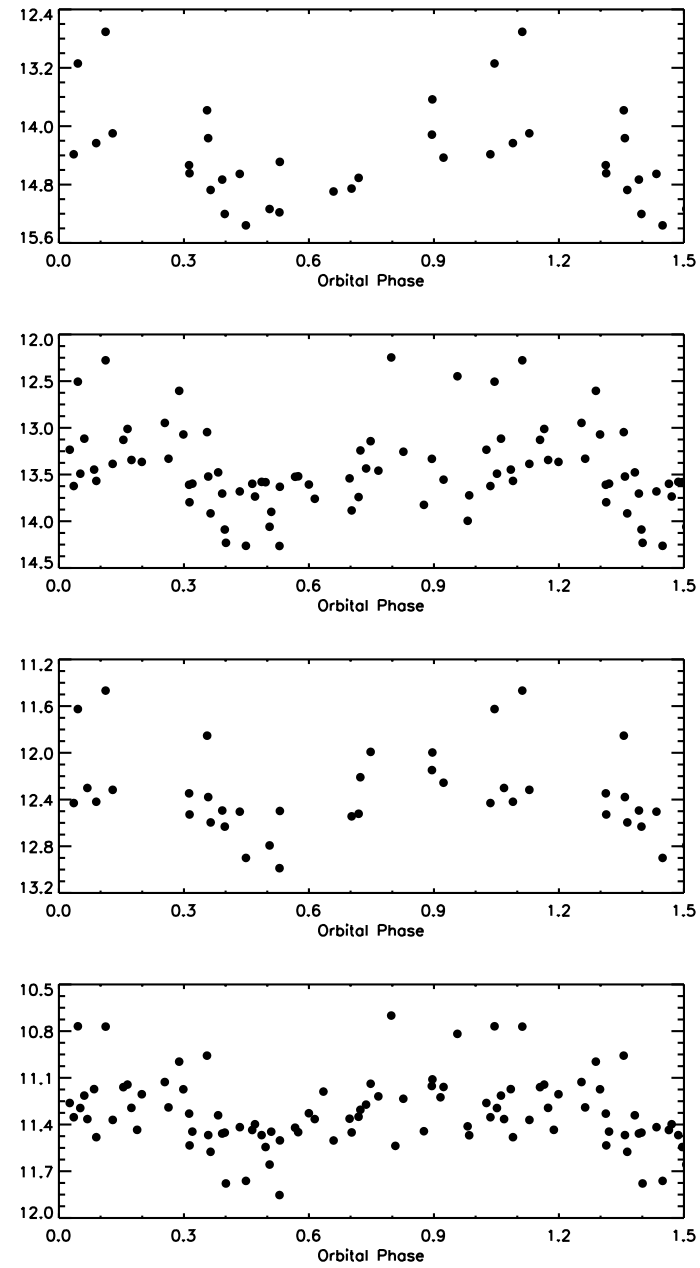
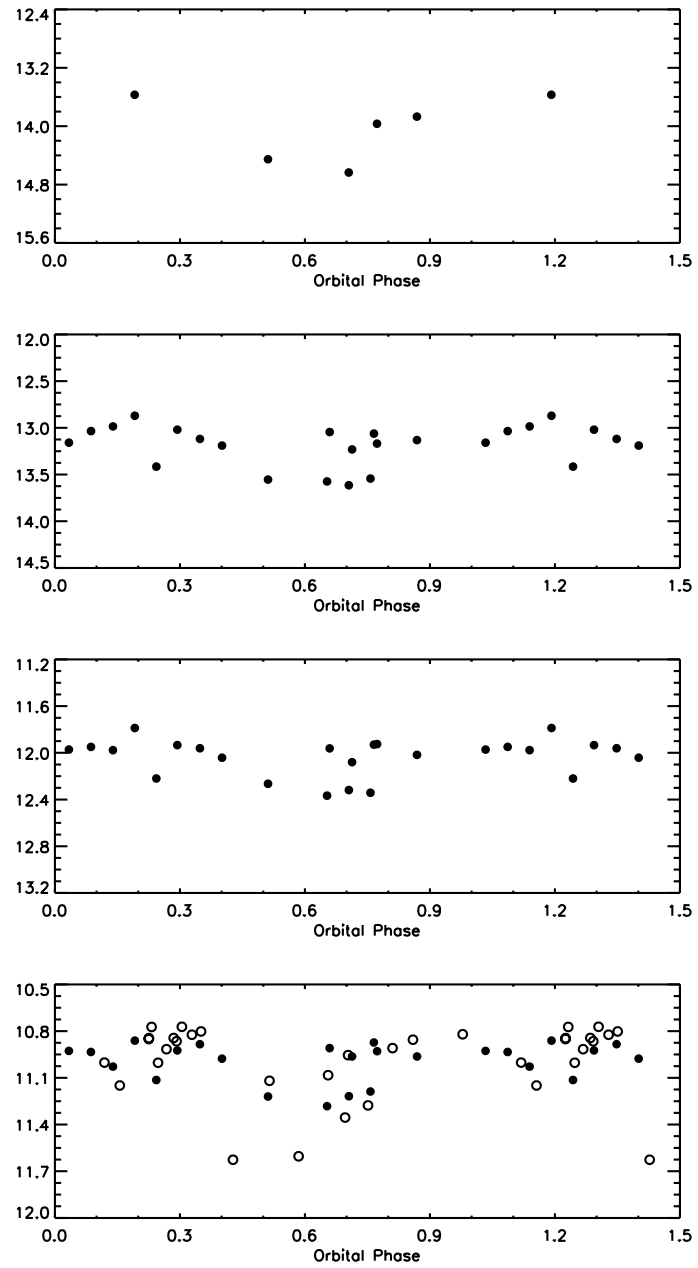

FIG. 5.-BVRI magnitudes for UZ Tau E folded at the binary orbital period and plotted against the binary orbital phase. Left panels: 2003-2004; right panels: 2005-2006. The open circles in the lower right panel show the VVO $I$-band data, which do not have corresponding $B, V$, and $R$ data.

in the spectroscopic data, since the two data sets differ in two important respects. First, the $\mathrm{H} \alpha$ data have much sparser sampling; they span a total of 8 years (1994-2002), but with only a handful of points during a given year. Second, they do not overlap at all with the photometric data. Given that the photometric data show both long-term trends and short-term scatter in addition to the periodic variations, and that $\mathrm{T}$ Tauri stars in general are known to show significant random variability at $\mathrm{H} \alpha$, it may be difficult to separate random and periodic variations (if any) without a dedicated monitoring campaign, preferably one that includes simultaneous photometric and spectroscopic measurements. We conclude that while periodic spectroscopic variations similar to those seen in the photometry are not definitively present in these spectroscopic data, neither are they ruled out.

\section{DISCUSSION}

The photometric data (and possibly the spectroscopic data) show periodic variability at the binary orbital period, suggesting that there is a link between the variability and interactions of the binary with its circumstellar and/or circumbinary material. In this section, we first argue that the periodic variations are unlikely to be due to stellar rotation, and then we examine how well the observed behavior matches what is expected from the pulsed accretion model of AL96. Finally, we examine the available data for other spectroscopic binaries to assess whether or not there is evidence for periodic accretion as a general phenomenon.

\subsection{Could the Variations Be Due to Rotation?}

Periodic variability is not uncommon in photometric studies of PMS stars. Indeed, dedicated monitoring surveys of rich starforming regions (e.g., Mandel \& Herbst 1991; Attridge \& Herbst 1992; Choi \& Herbst 1996; Stassun et al. 1999; Rebull 2001; Herbst et al. 2002a) have now discovered hundreds of PMS stars exhibiting periodic variability, the result of surface-brightness inhomogeneities (i.e., starspots) that rotate in and out of view with the stellar rotation period. The periodic variability observed in UZ Tau E is very unlikely to be the result of such rotationally modulated spot signals, for several reasons. First, the rotation periods of low-mass PMS stars are nearly always shorter than about 12 days, while the period of the variations reported here is 19 days. Among 150 low-mass PMS stars in the Orion Nebula Cluster, only two stars $(\sim 1 \%)$ have $P_{\text {rot }}>15$ days (Herbst \& Mundt 2005). Second, rotationally modulated spot signals are typically sinusoidal, and stable over many cycles or-in some cases - many years. In contrast, the periodic signal we have found in UZ Tau E is decidedly nonsinusoidal, with considerable scatter; the "bright" state has a duty cycle of $\sim 60 \%$. Thus, it is either intrinsically nonsinusoidal or shows substantial phase shifting from one cycle to the next; neither of these is consistent with rotationally modulated variability.

The rotation period distributions discussed above are presumably dominated by single stars or members of wide binaries, while tidal interactions between the stars in a close binary system 

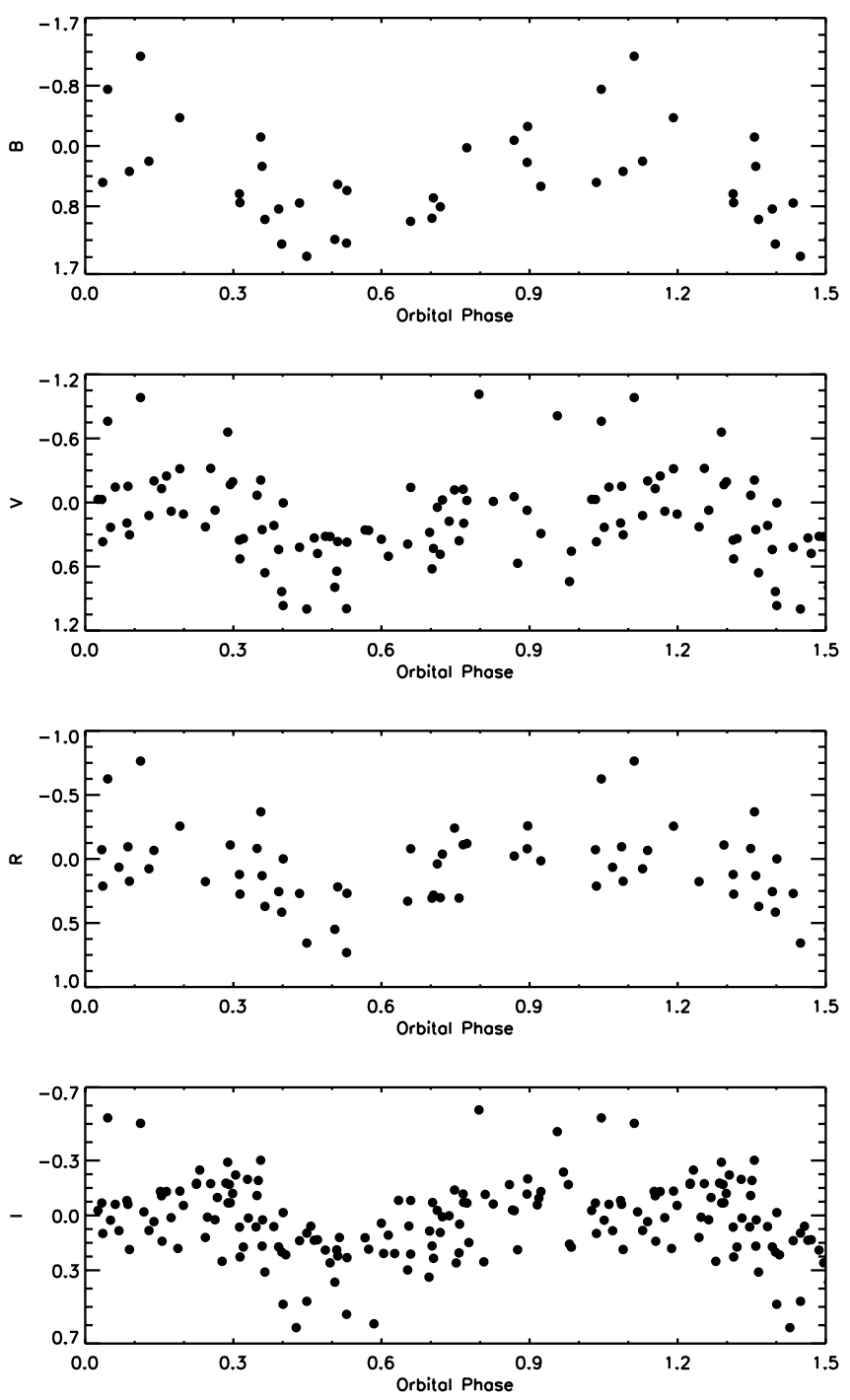

FIG. 6. - BVRI magnitudes for UZ Tau E folded at the binary orbital period and plotted against the binary orbital phase, after removing a long-term linear trend from each band.

can synchronize the orbital and rotational periods. However, in the case of eccentric systems like UZ Tau E, pseudosynchronization (in which the stellar angular velocity is synchronized with the orbital angular velocity at periastron) occurs instead, since the tidal interactions are strongest around periastron (Hut 1981). The predicted pseudosynchronous rotation period for UZ Tau E, using the weak friction formulation of Hut (1981) and the orbital parameters in Table 3, is $11.4 \pm 1.2$ days, inconsistent with the observed variability period.

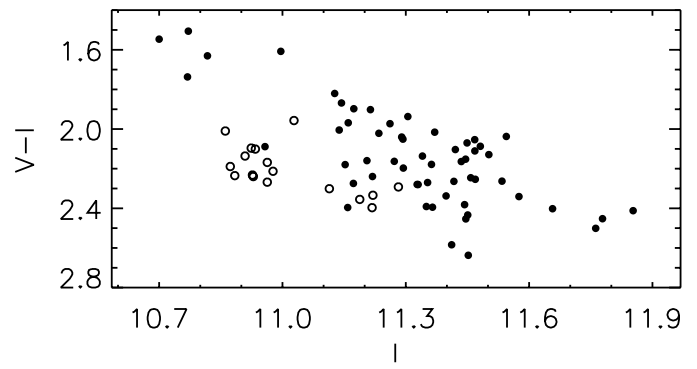

We can also estimate the rotation period of the UZ Tau E primary directly if three quantities are known: the inclination $i_{\text {rot }}$ of the star's rotation axis, the star's projected rotational velocity $v \sin i_{\text {rot }}$, and the stellar radius. Of these, the inclination is typically impossible to measure, except under special circumstances.

As noted in $\S 3$, the dynamical mass measurement of UZ Tau $\mathrm{E}$ allows us to determine the binary orbital inclination $i_{\text {orbit }}$. Based on studies of other binary systems, it is reasonable to assume that this inclination is the same as that of the stellar rotation axis, $i_{\text {rot }}$. The most detailed study comparing the orientations of these axes in binary systems is that of Hale (1994). Considering spectral types of F5-K5, he finds that binaries with separations less than 30-40 AU tend to exhibit coplanarity between rotational equators and orbital planes, while wider binaries have random orientations. Using a similar method, Weis (1974) found a tendency for the stellar rotational equators to align with the binary orbit among primaries in $\mathrm{F}$ star binaries. It is interesting to note that Weis (1974) did not find a tendency toward coplanarity between rotational and orbital planes among A stars, suggesting that caution is necessary when comparing stars of different masses. Similarly, Guthrie (1985) found no correlation between orbital inclination and $v \sin i$ among $23 \mathrm{~A} 2-\mathrm{A} 9$ binaries with semimajor axes of 10-70 AU. The low mass and short period of UZ Tau E suggest, however, that the conclusions of Hale (1994) are most applicable here.

Prato et al. (2002) found $L=0.63_{-0.17}^{+0.19} L_{\odot}$ and $T_{\text {eff }}=3700 \pm$ $150 \mathrm{~K}$ for the primary in UZ Tau E. Combining these values yields $R=1.9 \pm 0.2 R_{\odot}$. Hartmann \& Stauffer (1989) found $v \sin i=15.9 \pm 4.0 \mathrm{~km} \mathrm{~s}^{-1}$ for UZ Tau E using optical spectra, consistent with the value $v \sin i=16 \pm 2 \mathrm{~km} \mathrm{~s}^{-1}$, which we measure from our new spectra and adopt here. Since absorption lines of the secondary of UZ Tau E have only been seen in nearinfrared spectra and are not evident in any of our optical spectra, we take this to be the projected rotation velocity of the primary. Combining these measurements with $\sin i_{\text {orbit }}=0.81 \pm 0.05$ (§3), and assuming $i_{\text {orbit }}=i_{\text {rot }}$, we find $P_{\text {rot }}=4.9 \pm 0.8$ days. If $i_{\text {orbit }} \neq i_{\text {rot }}$, we find $P_{\text {rot }} \leq 6 \pm 1$ days, since $\sin i \leq 1$. Thus, uncertainty on the inclination cannot reconcile the photometric period with the inferred rotation period.

The most uncertain remaining quantity is $v \sin i$, but since Hartmann \& Stauffer (1989) measured $v \sin i$ from 11 different spectra of UZ Tau E, with self-consistent results from two different parts of the spectrum (including spectra near $\lambda=5200 \AA$ ) and consistency with our new $v \sin i$ measurement, it is unlikely that line broadening from photospheric lines of the faint, red secondary could lead to an overestimate of $v \sin i$ by a factor of 4 . Similarly, given the uncertainties on $L$ and $T_{\text {eff }}$, it is difficult to see how the radius could be underestimated by a factor of 4 . Thus, we conclude that the observed periodic variations are unlikely to be due to stellar rotation.

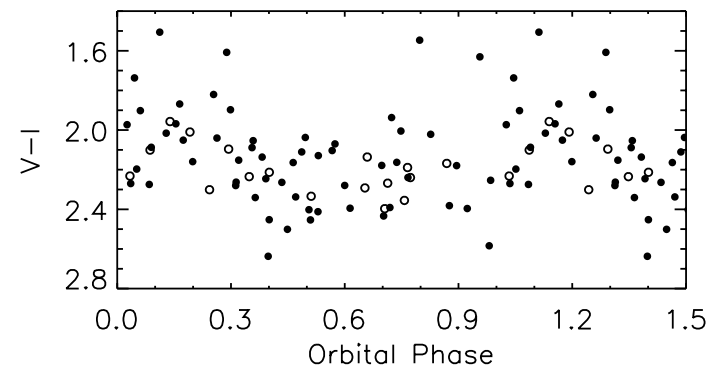

Fig. 7. $-V-I$ color vs. I magnitude and vs. orbital phase for 2003-2004 ( filled circles) and 2005-2006 (open circles). The system is redder when fainter and bluer when brighter, the expected behavior either for changes in extinction or for brightening due to increased accretion. 

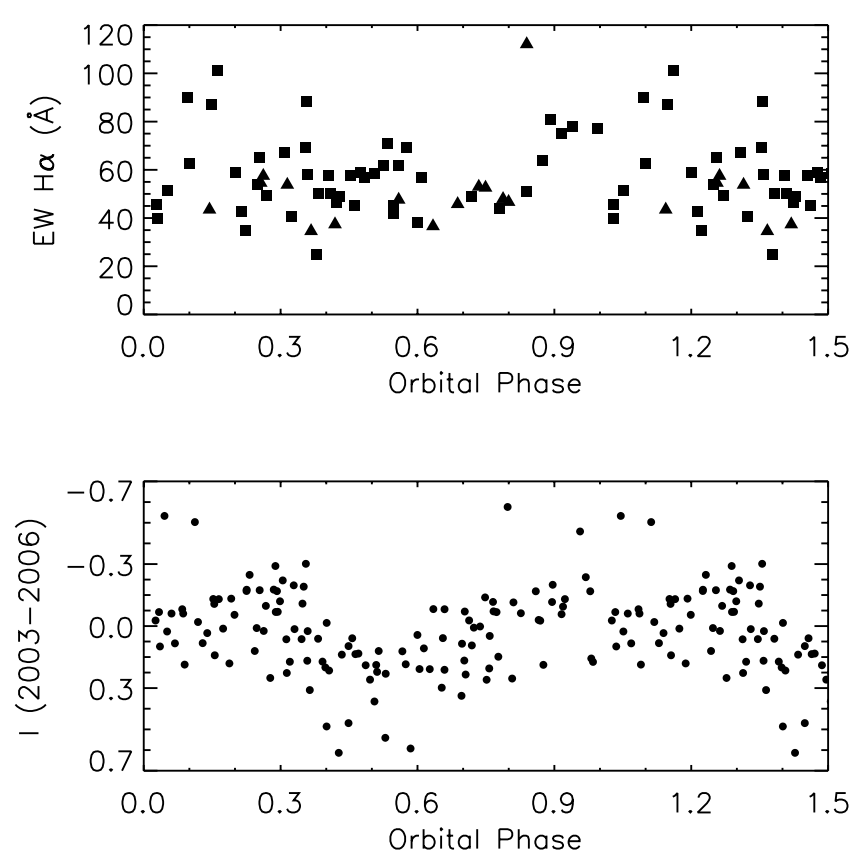

FIG. 8. - Top: Equivalent width of the $\mathrm{H} \alpha$ line as a function of binary orbital phase. Squares are our new measurements; triangles are from Martín et al. (2005). Bottom: For comparison, the phased I-band data. There is some suggestion of reduced $\mathrm{H} \alpha$ equivalent width at phases of $0.4-0.8$ as seen in the photometric data, but the data are too sparse there to provide clear evidence for periodic variability of the $\mathrm{H} \alpha$ emission.

\subsection{Evidence for Pulsed Accretion}

We have shown above that UZ Tau E exhibits periodic photometric variations that have the same period as the binary orbit and that these variations are unlikely to be caused by stellar rotation. Here we examine the predictions made by the pulsed accretion model of AL96 and compare them to our observations.

\subsubsection{What Are the Predictions?}

Broadly speaking, AL96 predicted that a binary with an eccentric orbit and a circumbinary disk would have an accretion flow from the circumbinary disk - and thus onto the circumstellar disks or stellar surfaces - that varies periodically at the binary orbital period.

The exact behavior of the accretion rate with orbital phase depends on the binary orbital parameters. AL96 showed the results of two simulations, one for mass ratio $M_{2} / M_{1}=0.43$ and eccentricity $e=0.1$, and another for $M_{2} / M_{1}=0.79$ and $e=0.5$. The former shows accretion that varies relatively smoothly over the orbital period, while the latter is strongly peaked at periastron.

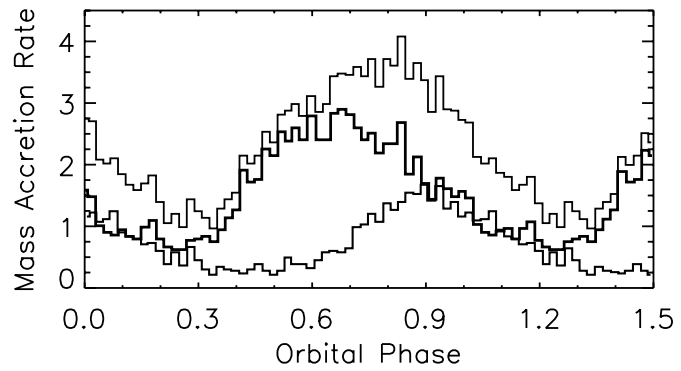

As noted by AL96, the exact timing of the accretion variability depends on the orbital parameters, most strongly on $e$. Some previous observational studies of $\mathrm{T}$ Tauri spectroscopic binaries have focused specifically on looking for enhanced accretion near periastron; however, we note here that the actual prediction of the model is more general than that, and that the peak accretion rate need not come near periastron.

\subsubsection{How Well Do the Data Match the Predictions?}

First, we note that our observations match the general predictions of AL96 quite well, in that there are indeed periodic photometric variations at the binary orbital period, which are readily interpretable as a variable accretion rate. The comparison with the spectroscopic data is more ambiguous; if more intensive monitoring of the $\mathrm{H} \alpha$ line in UZ Tau $\mathrm{E}$ were to show that there are no orbit-modulated $\mathrm{H} \alpha$ variations, it would present a problem for the model.

For a more specific comparison with our data, Figure 9 shows the variations of accretion with orbital phase predicted by AL96 for a binary with $M_{2} / M_{1}=0.43, e=0.1$. UZ Tau E has a more extreme mass ratio $\left(M_{2} / M_{1}=0.30\right)$ and larger eccentricity $(e=0.33)$ than this, but these parameters are closer to those of UZ Tau E than those of the other simulation in AL96. AL96 did note that the timing of the maxima of the accretion depends largely on $e$ rather than $M_{2} / M_{1}$. Since $e$ for UZ Tau E is intermediate between the two models calculated by AL96, we might then expect the maximum accretion to come between the phase of $\sim 0.75$ they calculate for the low-e case and the phase of $\sim 1$ for the high-e case.

For comparison with our data, we have taken the logarithm of the variations of accretion rate predicted by AL96 to shift them onto a "magnitude-like" scale, and added an arbitrary offset and scale factor to match the mean of the data and amplitude of the variations. The phase of the minimum predicted by this simulation does not match our data well; when the model is given a shift of +0.2 in orbital phase, there is better agreement between the model predictions and the data. This scaling and shifting to match the data is obviously ad hoc, but it allows us to compare the phase width of the observed variations, which appear to match the predictions relatively well. In addition, this shifted position of the maximum is indeed between the two cases calculated by AL96, as expected if eccentricity is the dominant factor in determining the timing of maximum accretion.

\subsection{Evidence for Periodic Accretion in Other T Tauri Binaries}

The discussion and data above show that looking for evidence of periodic accretion can be complicated, with other sources of variability perhaps being important and masking the effect in

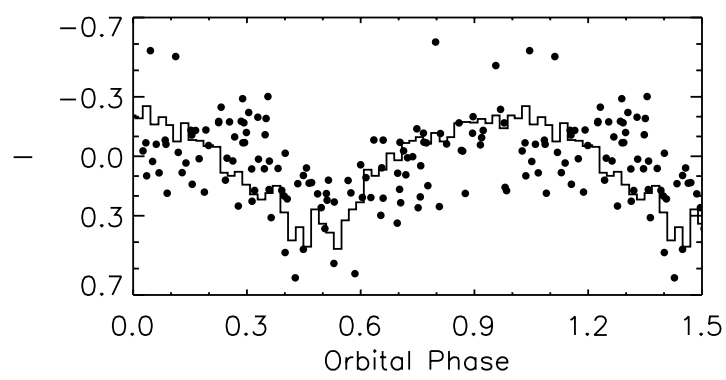

FIG. 9. - Left: Theoretical predictions of AL96 for the dependence of accretion rate on binary orbital phase in a binary with mass ratio $M_{2} / M_{1}=0.43, e=0.1$. The top curve shows the total accretion, while the lower curves show accretion onto the secondary (higher, thicker curve) and primary (lower, thinner curve). Right: Same total accretion curve, but placed onto a logarithmic scale and shifted vertically for comparison with the phased $I$-band data. The model here has been given an ad hoc shift of +0.2 in phase, roughly what is expected given the binary eccentricity (see $\S 5.2 .2$ ). 
TABLE 4

CTTS Spectroscopic Binaries

\begin{tabular}{|c|c|c|c|c|c|c|c|c|c|}
\hline Binary System & $\begin{array}{l}\text { Period } \\
\text { (days) }\end{array}$ & $e$ & $M_{2} / M_{1}$ & Spectral Type & $\begin{array}{c}L \\
\left(L_{\odot}\right)\end{array}$ & $\begin{array}{c}\text { Disk Mass }{ }^{\mathrm{a}} \\
\left(M_{\odot}\right)\end{array}$ & $\begin{array}{l}\text { Photometric } \\
\text { Periodicity? }\end{array}$ & $\begin{array}{l}\text { Balmer Line } \\
\text { Periodicity? }\end{array}$ & References \\
\hline V4046 Sgr....................... & 2.421 & $\leq 0.01$ & 0.94 & K5 & 0.82 & 0.0085 & $\operatorname{Yes}(\Delta B \approx 0.1)$ & Yes & $1,2,3$ \\
\hline GW Ori ........................... & 241.9 & 0.04 & SB1 & G0 & 26 & 0.3 & $?(\Delta V \approx 0.7)$ & $?$ & 4,5 \\
\hline UZ Tau E ...................... & 19.131 & 0.33 & 0.30 & M1 & 0.91 & 0.063 & $\operatorname{Yes}(\Delta I \approx 0.8)$ & Maybe & $6,7,8$ \\
\hline ROXs 43B...................... & 89.1 & 0.41 & SB1 & G0 & 0.4 & $<0.00037$ & $?(\Delta V=0.1)$ & $?$ & $1,9,10,11$ \\
\hline AK Sco .......................... & 13.609 & 0.47 & 0.99 & F5 & 8.40 & 0.002 & No $(\Delta y \approx 1.5)$ & Yes & $1,12,13$ \\
\hline ROXs 42 ......................... & 35.95 & 0.48 & 0.92 & K4 & 0.4 & $<0.00025$ & $?(\Delta V=0.4)$ & $?$ & $1,10,11,14,15$ \\
\hline DQ Tau ............................ & 15.804 & 0.56 & 0.97 & K7-M1 & 0.95 & 0.020 & $\operatorname{Yes}(\Delta V \approx 0.5)$ & Yes & 16,17 \\
\hline 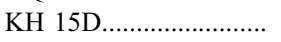 & 48.38 & $0.57-0.65$ & $0.83^{\mathrm{b}}$ & $\mathrm{K} 7^{\mathrm{c}}$ & $0.4^{\mathrm{c}}$ & $\ldots$ & Eclipse $(\Delta I \approx 3.5)$ & $?$ & $18,19,20$ \\
\hline
\end{tabular}

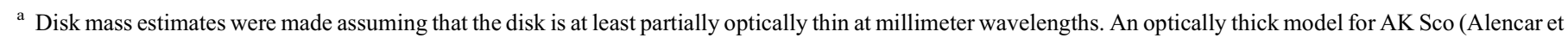
al. 2003) yields a disk mass of $0.02 M_{\odot}$.

${ }^{\mathrm{b}}$ Derived from the stellar luminosity ratio that best fits the eclipse data (Winn et al. 2006).

c Properties of the secondary star, since the primary is never visible.

References.- (1) Jensen \& Mathieu 1997; (2) Quast et al. 2000; (3) Mekkaden 2000; (4) Mathieu et al. 1991; (5) Mathieu et al. 1995; (6) This work; (7) Prato et al. 2002;

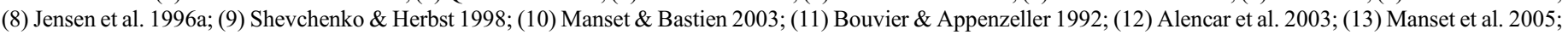
(14) Lee 1992; (15) Walter et al. 1994; (16) Mathieu et al. 1997; (17) Basri et al. 1997; (18) Hamilton et al. 2001; (19) Hamilton et al. 2005 ; (20) Winn et al. 2006.

small data sets, and with the exact behavior expected to be a function of the specific binary orbital parameters. That said, is evidence for pulsed accretion seen in other young binary systems? In Table 4 we present characteristics of young binaries with periods of less than $1 \mathrm{yr}$ and evidence of circumbinary material, in order of increasing eccentricity. Below, we examine the observational data for some of these systems, attempting to relate them to what we see in UZ Tau and exploring similarities and differences. Unfortunately, the small number of systems and their somewhat heterogeneous properties means that it is difficult to generalize, so we offer these comments in the spirit of attempting to pull together the existing data, rather than arguing one way or the other for the validity of the AL96 model for the sample as a whole.

\subsection{1. $D Q T a u$}

DQ Tau was the first system to be scrutinized for evidence of pulsed accretion. Mathieu et al. (1997) showed that the photometric variations are modulated at the binary orbital period, and Basri et al. (1997) showed that the $\mathrm{H} \alpha$ line and spectral veiling are as well. Fortuitously, the mass ratio and eccentricity of DQ Tau are quite similar to those of the high-eccentricity case modeled by AL96, allowing for specific comparison with the theory. The timing and phase width of the photometric and spectroscopic variations match the predictions well, being sharply peaked near periastron. However, the DQ Tau observations did show considerable orbit-to-orbit variation, with the periastron brightening being seen roughly $65 \%$ of the time. This is reminiscent of the large scatter that we see in the UZ Tau light curves; clearly the periodic accretion process is not exactly repeatable from one orbit to the next, nor is it the only source of variability.

\subsection{2. $A K S c o$}

The orbital eccentricity and binary mass ratio of AK Sco are quite similar to those of DQ Tau, and indeed, simulations by Günther \& Kley (2002) for a binary with AK Sco's orbital parameters predict pulsed accretion. Thus, it comes as some surprise that the system does not show periodic photometric variability, despite extensive monitoring (Alencar et al. 2003). The overall variability is large (up to $1.5 \mathrm{mag}$ in $y$ ) but apparently random. There are periodic variations in the Balmer lines, but they are not sharply peaked around periastron. Examining Table 4, we note two properties of AK Sco that are quite different from those of DQ Tau or UZ Tau. First, AK Sco is considerably hotter and more luminous. Thus, accretion variations of the same luminosity as those occurring in UZ Tau and DQ Tau would result in substantially smaller magnitude changes, which could be swamped by the large random variability. Second, AK Sco has considerably lower millimeter flux than either of the other two systems. If the systems are fit with similar disk models (in which the disk is assumed to be optically thin at millimeter wavelengths in its outer regions), AK Sco's disk mass is an order of magnitude smaller than that of DQ Tau or UZ Tau E (Jensen et al. 1996a; Jensen \& Mathieu 1997; Mathieu et al. 1997). Alencar et al. (2003) fit AK Sco with an optically thick disk model that has a comparable mass to the disk models fit to DQ Tau and UZ Tau E. However, such disk models have not been fit to DQ Tau or UZ Tau E and would presumably result in even larger disk masses for those systems. In a direct comparison of $\lambda=1.1 \mathrm{~mm}$ flux, DQ Tau and UZ Tau E are 3 and 5 times brighter than AK Sco at roughly the same distance, presumably reflecting larger disk masses. It is possible that a somewhat lower mass disk has different dynamics and that the accretion flow in the AK Sco system is fundamentally different than that in the other systems with more massive disks.

\subsubsection{GW Ori}

This system has a near-circular orbit and thus would not be expected to show pulsed accretion under the model set forth by AL96. However, Stempels \& Gahm (2004) quoted a private communication from P. Artymowicz as saying that pulsed accretion is possible for systems with circular orbits as well, and indeed D'Angelo et al. (2006) showed that this occurs for giant planets embedded in disks. Thus, pulsed accretion appears to be possible for at least some circular-orbit systems and thus may be for GW Ori as well, although the larger gap cleared by a stellar companion (Artymowicz \& Lubow 1994) will clear some of the disk resonances that might contribute to disk eccentricity growth in a system with a planetary-mass companion.

Like AK Sco, GW Ori is very luminous and shows significant random variability, although no obvious periodic variability. It does have a much more massive disk than AK Sco, however, and indeed more massive than any of the systems considered here. Because of its much larger semimajor axis, and to some 
extent its circular orbit, GW Ori is much more likely to have significant circumstellar disks, as the stars do not approach each other very closely at periastron. Thus, material flowing from the circumbinary disk may merge with the circumstellar disks and then accrete more gradually onto the stars, rather than falling directly on (or near) the stellar surfaces, as is expected to happen in the shorter period systems. If the infalling material does not shock strongly as it merges with the circumstellar disk, and if any density enhancements are smoothed out somewhat by the time the material reaches the stellar surface, then any photometric signature of the periodic infall would be weakened. We note that UZ Tau E likely has circumstellar disks as well (Jensen et al. 1996a), so a similar effect could be at work in reducing the amplitude of the periodic variability relative to the stochastic variability.

\subsubsection{V4046 Sgr}

Like GW Ori, V4046 Sgr has a nearly circular orbit. However, V4046 Sgr has shown periodic photometric variations at the binary orbital period (Quast et al. 2000; Mekkaden 2000). These variations persist over several years and are relatively sinusoidal (F. M. Walter 2003-2005, unpublished). Unlike the other binaries discussed here, in this case stellar rotation is a plausible explanation of the observed variations. It is common for stellar rotational periods to become synchronized with the binary orbital period, particularly for short-period binaries like V4046 Sgr. Given the short period (resulting in stronger tidal interactions and a shorter synchronization timescale) and the somewhat older age of this system ( $\sim 10 \mathrm{Myr})$, synchronization is plausible and indeed is supported by detailed analysis of the system (Stempels \& Gahm 2004). However, rotation does not explain the periodic Balmer line variations observed, which Stempels \& Gahm (2004) attribute to accumulations of gas corotating with the binary orbit.

\subsubsection{ROXS 42 and ROXS $43 B$}

These two spectroscopic binaries are both weak-lined T Tauri stars (Bouvier \& Appenzeller 1992; Walter et al. 1994), indicating less-active accretion than some of the other systems discussed here. Neither has been detected at millimeter wavelengths, yielding only an upper limit on the disk masses (Skinner et al. 1991; Jensen et al. 1996b). Both systems show mid-infrared excesses, indicating the presence of circumbinary material, and a lack of near-infrared excess, which can be modeled as a cleared central region in the disk (Jensen \& Mathieu 1997). The fact that both are higher order multiple systems complicates matters; ROXs 42 (NTTS 162814-2427) is a triple system with a separation of $0.15^{\prime \prime}$ (Lee 1992; Ghez et al. 1993), while ROXs 43B (NTTS 162819-2423S) has a wide companion at $4.8^{\prime \prime}$, which is itself a close binary system (Walter et al. 1994; Simon et al. 1995b). Since the evidence for the presence of a substantial disk rests on the low-spatial-resolution IRAS detections, it is possible that the excess is associated with the wider companions rather than arising from circumbinary disks around the spectroscopic binaries. In any case, the lack of millimeter detections indicates that there is less disk mass in these two systems than in the others discussed here. Neither system has been intensively monitored over time spans that would be necessary to detect periodic photometric variations at the relatively long orbital periods. ROXs 42 shows evidence for some semiregular variations over roughly 1.5 orbital periods (Zakirov et al. 1993), while the combined light of the ROX 43 system shows only a 0.1 mag variation, with evidence of a 1.5 day or 3 day periodicity, presumably attributable to rotation of one or more of the stars (Shevchenko \& Herbst 1998).

\subsection{6. $K H 15 D$}

The unusual pre-main-sequence system KH 15D (V582 Mon) is a spectroscopic binary that undergoes deep $(\Delta I \sim 3.5 \mathrm{mag})$ eclipses, thought to arise due to occultation from a circumbinary disk (Hamilton et al. 2001, 2005; Herbst et al. 2002b; Winn et al. 2006 and references therein). While the system has an eccentricity and mass ratio that would suggest that pulsed accretion might be present, the photometric variations at the binary orbital period are dominated by the deep eclipses. Furthermore, the depth and detailed shape of these eclipses are evolving with time (Winn et al. 2003, 2006; Johnson \& Winn 2004; Maffei et al. 2005; Johnson et al. 2005), making it very difficult to determine whether there might currently be an additional, smaller amplitude component with the same period that is related to accretion rather than occultation. Winn et al. (2003) showed that the current deep eclipses did not occur during the first half of the twentieth century, raising the possibility of searching for evidence of accretionrelated variability at earlier epochs. Their limit of 1 mag on the variability during that time does not preclude accretion-related variations such as those seen in UZ Tau E. The $\sim 0.9$ mag periodic variations seen from the 1960s through the 1980s (Johnson \& Winn 2004; Maffei et al. 2005; Johnson et al. 2005), however, are relatively smooth and are well fit by the eclipse model (Winn et al. 2006), placing a limit on how much any accretion-related component was contributing to the variability during that time. Since the inferred mass ratio and eccentricity for KH $15 \mathrm{D}$ are similar to those of DQ Tau (Table 4), we might expect accretionrelated variability to be strongly peaked around periastron, which is also when the current deep eclipses occur. This might help explain several anomalously bright points seen during eclipses in the late 1990s that are not well fit by the model of Winn et al. (2006).

The precessing circumbinary disk occultation model of Winn et al. (2006) is quite successful in reproducing the shape and ongoing evolution of the light curve, and we do not suggest that accretion explains most of the photometric variations. We note, however, the possibility that such an additional component might be sporadically present (with the same period) and that, if it is, this could complicate the modeling of the historical evolution of the light curve, especially during earlier, more sparsely sampled epochs.

\section{CONCLUSIONS}

We have shown that the pre-main-sequence binary UZ Tau E shows clear photometric variability at the binary orbital period of 19.13 days. This variability is consistent with a model in which material in the circumbinary disk is periodically perturbed by the binary in its eccentric orbit and falls from the outer disk, across the cleared central gap and onto the stars or their circumstellar disks. There is significant scatter in the light curves, indicating that this "pulsed accretion" may not occur during every binary orbit. $\mathrm{H} \alpha$ equivalent widths show some suggestion of periodic variability, but it is not definitive.

The apparently intermittent behavior of the accretion, and the presence of other, random sources of variability, suggest that searches for this sort of accretion signature require well-sampled data sets with long time baselines in order to detect any periodic component. In particular, simultaneous photometric and spectroscopic monitoring of UZ Tau E in the future will help determine whether the $\mathrm{H} \alpha$ variations show a periodic component, as the photometric variations do.

The good overall agreement between theory and observations suggests that resonant interactions between stars (and, by 
extension, planets) and disks are indeed important in determining disk structure and dynamics, while the random component of the observed behavior shows that there is still work to be done in understanding the full complexity of these interactions.

We gratefully acknowledge the support of the National Science Foundation through grant AST 03-07830. We thank the referee, Steve Lubow, for useful comments that improved this paper. We are grateful to Michael Meyer, David Cohen, and Larry Marschall for useful discussions; to Marcos Huerta and Pat Hartigan for use of their spectra of UZ Tau E; to Matthew Richardson for assistance with data reduction; to Peter Collings for translating early papers on UZ Tau from German to English; and to Thierry Forveille for use of his ORBIT code. M. S. and F. W. are grateful for Stony Brook University's partial support of their participation in the SMARTS consortium. This research has made use of the SIMBAD database, operated at CDS, Strasbourg, France, and of NASA's Astrophysics Data System.
Alencar, S. H. P., \& Basri, G. 2000, AJ, 119, 1881

Alencar, S. H. P., Melo, C. H. F., Dullemond, C. P., Andersen, J., Batalha, C., Vaz, L. P. R., \& Mathieu, R. D. 2003, A\&A, 409, 1037

Artymowicz, P., \& Lubow, S. H. 1994, ApJ, 421, 651 1996, ApJ, 467, L77 (AL96)

Attridge, J. M., \& Herbst, W. 1992, ApJ, 398, L61

Bailey, S. I. 1921, Harvard Coll. Obs. Bull., 759, 1

Basri, G., Johns-Krull, C. M., \& Mathieu, R. D. 1997, AJ, 114, 781

Basri, G., \& Reiners, A. 2006, AJ, 132, 663

Bate, M. R., Bonnell, I. A., Clarke, C. J., Lubow, S. H., Ogilvie, G. I., Pringle, J. E., \& Tout, C. A. 2000, MNRAS, 317, 773

Beckwith, S. V. W., Sargent, A. I., Chini, R. S., \& Güsten, R. 1990, AJ, 99, 924

Bohlin, K. 1923, Astron. Nachr., 218, 203

Bouvier, J., \& Appenzeller, I. 1992, A\&AS, 92, 48

Byrne, P. B. 1986, Irish Astron. J., 17, 294

Choi, P. I., \& Herbst, W. 1996, AJ, 111, 283

Clarke, C. 1992, in ASP Conf. Ser. 32, Complementary Approaches to Double and Multiple Star Research, ed. H. A. McAlister \& W. I. Hartkopf (San Francisco: ASP), 176

D’Angelo, G., Lubow, S. H., \& Bate, M. R. 2006, ApJ, 652, 1698

Dutrey, A., Guilloteau, S., Duvert, G., Prato, L., Simon, M., Schuster, K., \& Menard, F. 1996, A\&A, 309, 493

Eggenberger, A., Udry, S., \& Mayor, M. 2004, A\&A, 417, 353

Forveille, T., et al. 1999, A\&A, 351, 619

Ghez, A. M., Neugebauer, G., \& Matthews, K. 1993, AJ, 106, 2005

Gudehus, D. H. 2001, BAAS, 33, 850

Günther, R., \& Kley, W. 2002, A\&A, 387, 550

Guthrie, B. N. G. 1985, MNRAS, 215, 545

Hale, A. 1994, AJ, 107, 306

Hamilton, C. M., Herbst, W., Shih, C., \& Ferro, A. J. 2001, ApJ, 554, L201

Hamilton, C. M., et al. 2005, AJ, 130, 1896

Hartmann, L., \& Stauffer, J. R. 1989, AJ, 97, 873

Herbig, G. H. 1977, ApJ, 217, 693

Herbst, W., Bailer-Jones, C. A. L., Mundt, R., Meisenheimer, K., \& Wackermann, R. 2002a, A\&A, 396, 513

Herbst, W., Herbst, D. K., Grossman, E. J., \& Weinstein, D. 1994, AJ, 108, 1906

Herbst, W., \& Mundt, R. 2005, ApJ, 633, 967

Herbst, W., et al. 2002b, PASP, 114, 1167

Horne, J. H., \& Baliunas, S. L. 1986, ApJ, 302, 757

Huerta, M., Hartigan, P., \& White, R. J. 2005, AJ, 129, 985

Hut, P. 1981, A\&A, 99, 126

Jensen, E. L. N., Koerner, D. W., \& Mathieu, R. D. 1996a, AJ, 111, 2431

Jensen, E. L. N., \& Mathieu, R. D. 1997, AJ, 114, 301

Jensen, E. L. N., Mathieu, R. D., Donar, A. X., \& Dullighan, A. 2004, ApJ, 600,789

Jensen, E. L. N., Mathieu, R. D., \& Fuller, G. A. 1994, ApJ, 429, L29 1996b, ApJ, 458, 312

Johnson, J. A., \& Winn, J. N. 2004, AJ, 127, 2344

Johnson, J. A., et al. 2005, AJ, 129, 1978

Joy, A. H., \& van Biesbroeck, G. 1944, PASP, 56, 123

Kenyon, S. J., Dobrzycka, D., \& Hartmann, L. 1994, AJ, 108, 1872

Kovacs, G. 1981, Ap\&SS, 78, 175

Larwood, J. D., Nelson, R. P., Papaloizou, J. C. B., \& Terquem, C. 1996, MNRAS, 282, 597

Lee, C.-W. 1992, Ph.D. thesis, Univ. Wisconsin, Madison

Lin, D. N. C., \& Papaloizou, J. C. B. 1993, in Protostars and Planets III, ed. E. H. Levy \& J. I. Lunine (Tucson: Univ. Arizona Press), 749
Lubow, S. H., \& Ogilvie, G. I. 2000, ApJ, 538, 326 2001, ApJ, 560, 997

Maffei, P., Ciprini, S., \& Tosti, G. 2005, MNRAS, 357, 1059

Mandel, G. N., \& Herbst, W. 1991, ApJ, 383, L75

Manset, N., \& Bastien, P. 2003, AJ, 125, 3274

Manset, N., Bastien, P., \& Bertout, C. 2005, AJ, 129, 480

Martín, E. L., Magazzù, A., Delfosse, X., \& Mathieu, R. D. 2005, A\&A, 429, 939

Mathieu, R. D., Adams, F. C., Fuller, G. A., Jensen, E. L. N., Koerner, D. W., \& Sargent, A. I. 1995, AJ, 109, 2655

Mathieu, R. D., Adams, F. C., \& Latham, D. W. 1991, AJ, 101, 2184

Mathieu, R. D., Baraffe, I., Simon, M., Stassun, K. G., \& White, R. 2007, in Protostars and Planets V, ed. B. Reipurth, D. Jewitt, \& K. Kei (Tucson: Univ. Arizona Press), 411

Mathieu, R. D., Martin, E. L., \& Magazzu, A. 1996, BAAS, 28, 920

Mathieu, R. D., Stassun, K., Basri, G., Jensen, E. L. N., Johns-Krull, C. M., Valenti, J. A., \& Hartmann, L. W. 1997, AJ, 113, 1841

Mekkaden, M. V. 2000, in IAU Symp. 200, The Formation of Binary Stars, ed. B. Reipurth \& H. Zinnecker (San Francisco: ASP), 31

Monet, D. G., et al. 2003, AJ, 125, 984

Monin, J. L., Clarke, C. J., Prato, L., \& McCabe, C. 2007, in Protostars and Planets V, ed. B. Reipurth, D. Jewitt, \& K. Kei (Tucson: Univ. Arizona Press), 395

Osterloh, M., \& Beckwith, S. V. W. 1995, ApJ, 439, 288

Ostriker, E. C., Shu, F. H., \& Adams, F. C. 1992, ApJ, 399, 192

Papaloizou, J. C. B., \& Terquem, C. 1995, MNRAS, 274, 987

Prato, L., Simon, M., Mazeh, T., Zucker, S., \& McLean, I. S. 2002, ApJ, 579, L99

Quast, G. R., Torres, C. A. O., de La Reza, R., da Silva, L., \& Mayor, M. 2000, in IAU Symp. 200, The Formation of Binary Stars, ed. B. Repurth \& H. Zinnecker (San Francisco: ASP), 28

Rebull, L. M. 2001, AJ, 121, 1676

Scargle, J. D. 1982, ApJ, 263, 835

Schwarzenberg-Czerny, A. 1989, MNRAS, 241, 153

Shevchenko, V. S., \& Herbst, W. 1998, AJ, 116, 1419

Simon, M., Chen, W. P., Howell, R. R., Benson, J. A., \& Slowik, D. 1992, ApJ, 384,212

Simon, M., Dutrey, A., \& Guilloteau, S. 2000, ApJ, 545, 1034

Simon, M., et al. 1995a, ApJ, 443, 625 1995b, ApJ, 443, 625

Skinner, S. L., Brown, A., \& Walter, F. M. 1991, AJ, 102, 1742

Stassun, K. G., Mathieu, R. D., Mazeh, T., \& Vrba, F. J. 1999, AJ, 117, 2941

Stellingwerf, R. F. 1978, ApJ, 224, 953

Stempels, H. C., \& Gahm, G. F. 2004, A\&A, 421, 1159

Walter, F. M., Brown, A., Mathieu, R. D., Myers, P. C., \& Vrba, F. J. 1988, AJ, 96, 297

Walter, F. M., Vrba, F. J., Mathieu, R. D., Brown, A., \& Myers, P. C. 1994, AJ, 107,692

Weis, E. W. 1974, ApJ, 190, 331

Winn, J. N., Garnavich, P. M., Stanek, K. Z., \& Sasselov, D. D. 2003, ApJ, 593, L121

Winn, J. N., Hamilton, C. M., Herbst, W. J., Hoffman, J. L., Holman, M. J., Johnson, J. A., \& Kuchner, M. J. 2006, ApJ, 644, 510

Zakirov, M. M., Azimov, A. A., \& Grankin, K. N. 1993, Inf. Bull. Variable Stars, 3898, 1 